\title{
\begin{tabular}{l|l} 
Mibraries & DSpace@MIT
\end{tabular}
}

MIT Open Access Articles

Search for High-Mass Resonances Decaying to Dimuons at CDF

The MIT Faculty has made this article openly available. Please share how this access benefits you. Your story matters.

Citation: CDF Collaboration et al. “Search for High-Mass Resonances Decaying to Dimuons at CDF." Physical Review Letters 102.9 (2009): 091805. (C) 2009 The American Physical Society

As Published: http://dx.doi.org/10.1103/PhysRevLett.102.091805

Publisher: American Physical Society

Persistent URL: http://hdl.handle.net/1721.1/52313

Version: Final published version: final published article, as it appeared in a journal, conference proceedings, or other formally published context

Terms of Use: Article is made available in accordance with the publisher's policy and may be subject to US copyright law. Please refer to the publisher's site for terms of use. 


\section{Search for High-Mass Resonances Decaying to Dimuons at CDF}

T. Aaltonen, ${ }^{24}$ J. Adelman, ${ }^{14}$ T. Akimoto, ${ }^{56}$ B. Álvarez González, ${ }^{12}$ S. Amerio, ${ }^{44 b, 44 a}$ D. Amidei,${ }^{35}$ A. Anastassov, ${ }^{39}$ A. Annovi ${ }^{20}$ J. Antos, ${ }^{15}$ G. Apollinari,${ }^{18}$ A. Apresyan, ${ }^{49}$ T. Arisawa, ${ }^{58}$ A. Artikov, ${ }^{16}$ W. Ashmanskas, ${ }^{18}$ A. Attal, ${ }^{4}$ A. Aurisano, ${ }^{54}$ F. Azfar, ${ }^{43}$ P. Azzurri, ${ }^{47 \mathrm{~d}, 47 \mathrm{a}}$ W. Badgett, ${ }^{18}$ A. Barbaro-Galtieri, ${ }^{29}$ V. E. Barnes, ${ }^{49}$ B. A. Barnett, ${ }^{26}$ V. Bartsch, ${ }^{31}$ G. Bauer, ${ }^{33}$ P.-H. Beauchemin, ${ }^{34}$ F. Bedeschi, ${ }^{47 a}$ D. Beecher,${ }^{31}$ S. Behari, ${ }^{26}$ G. Bellettini, ${ }^{47 b, 47 a}$ J. Bellinger, ${ }^{60}$ D. Benjamin, ${ }^{17}$ A. Beretvas, ${ }^{18}$ J. Beringer, ${ }^{29}$ A. Bhatti, ${ }^{51}$ M. Binkley, ${ }^{18}$ D. Bisello, ${ }^{44 b, 44 a}$ I. Bizjak,${ }^{31, w}$ R. E. Blair, ${ }^{2}$ C. Blocker, ${ }^{7}$ B. Blumenfeld, ${ }^{26}$ A. Bocci,${ }^{17}$ A. Bodek,${ }^{50}$ V. Boisvert, ${ }^{50}$ G. Bolla, ${ }^{49}$ D. Bortoletto, ${ }^{49}$ J. Boudreau, ${ }^{48}$ A. Boveia, ${ }^{11}$ B. Brau, ${ }^{11, b}$ A. Bridgeman, ${ }^{25}$ L. Brigliadori, ${ }^{44 a}$ C. Bromberg, ${ }^{36}$ E. Brubaker, ${ }^{14}$ J. Budagov, ${ }^{16}$ H. S. Budd, ${ }^{50}$ S. Budd,${ }^{25}$ S. Burke, ${ }^{18}$ K. Burkett, ${ }^{18}$ G. Busetto, ${ }^{44 b, 44 a}$ P. Bussey,${ }^{22,1}$ A. Buzatu, ${ }^{34}$ K. L. Byrum, ${ }^{2}$ S. Cabrera, ${ }^{17, u}$ C. Calancha, ${ }^{32}$ M. Campanelli, ${ }^{36}$ M. Campbell,,${ }^{35}$ F. Canelli, ${ }^{18}$ A. Canepa,${ }^{46}$ B. Carls, ${ }^{25}$ D. Carlsmith, ${ }^{60}$ R. Carosi,${ }^{47 a}$ S. Carrillo, ${ }^{19, \mathrm{n}}$ S. Carron, ${ }^{34}$ B. Casal, ${ }^{12}$ M. Casarsa, ${ }^{18}$ A. Castro, ${ }^{6 b, 6 a}$ P. Catastini, ${ }^{47 c, 47 a}$ D. Cauz, ${ }^{55 b, 55 a}$ V. Cavaliere, ${ }^{47 c, 47 a}$ M. Cavalli-Sforza ${ }^{4}$ A. Cerri, ${ }^{29}$ L. Cerrito, ${ }^{31, o}$ S. H. Chang, ${ }^{28}$ Y. C. Chen, ${ }^{1}$ M. Chertok, ${ }^{8}$ G. Chiarelli, ${ }^{47 a}$ G. Chlachidze, ${ }^{18}$ F. Chlebana, ${ }^{18}$ K. Cho, ${ }^{28}$ D. Chokheli, ${ }^{16}$ J. P. Chou, ${ }^{23}$ G. Choudalakis, ${ }^{33}$ S. H. Chuang, ${ }^{53}$ K. Chung, ${ }^{13}$ W. H. Chung, ${ }^{60}$ Y. S. Chung, ${ }^{50}$ T. Chwalek,${ }^{27}$ C. I. Ciobanu, ${ }^{45}$ M. A. Ciocci, ${ }^{47 c, 47 a}$ A. Clark, ${ }^{21}$ D. Clark,${ }^{7}$ G. Compostella, ${ }^{44 a}$ M. E. Convery, ${ }^{18}$ J. Conway, ${ }^{8}$ M. Cordelli, ${ }^{20}$ G. Cortiana, ${ }^{44 b, 44 a}$ C. A. Cox,${ }^{8}$ D. J. Cox ${ }^{8}$ F. Crescioli, ${ }^{47 b, 47 a}$

C. Cuenca Almenar, ${ }^{8, v}$ J. Cuevas, ${ }^{12, \mathrm{~s}}$ R. Culbertson, ${ }^{18}$ J. C. Cully, ${ }^{35}$ D. Dagenhart, ${ }^{18}$ M. Datta, ${ }^{18}$ T. Davies, ${ }^{22}$ P. de Barbaro, ${ }^{50}$ S. De Cecco, ${ }^{52 a}$ A. Deisher ${ }^{29}$ G. De Lorenzo, ${ }^{4}$ M. Dell'Orso, ${ }^{47 b, 47 a}$ C. Deluca, ${ }^{4}$ L. Demortier,${ }^{51}$ J. Deng, ${ }^{17}$ M. Deninno, ${ }^{6 a}$ P. F. Derwent, ${ }^{18}$ G. P. di Giovanni, ${ }^{45}$ C. Dionisi, ${ }^{52 b, 52 a}$ B. Di Ruzza, ${ }^{55 b, 55 a}$ J. R. Dittmann, ${ }^{5}$ M. D’Onofrio, ${ }^{4}$ S. Donati, ${ }^{47 b, 47 a}$ P. Dong, ${ }^{9}$ J. Donini, ${ }^{44 a}$ T. Dorigo, ${ }^{44 a}$ S. Dube,${ }^{53}$ J. Efron, ${ }^{40}$ A. Elagin, ${ }^{54}$ R. Erbacher, ${ }^{8}$ D. Errede, ${ }^{25}$ S. Errede,${ }^{25}$ R. Eusebi, ${ }^{18}$ H. C. Fang, ${ }^{29}$ S. Farrington, ${ }^{43}$ W. T. Fedorko, ${ }^{14}$ R. G. Feild, ${ }^{61}$ M. Feindt, ${ }^{27}$ J. P. Fernandez,${ }^{32}$ C. Ferrazza, ${ }^{47 d, 47 a}$ R. Field, ${ }^{19}$ G. Flanagan,${ }^{49}$ R. Forrest,${ }^{8}$ M. J. Frank, ${ }^{5}$ M. Franklin, ${ }^{23}$ J. C. Freeman, ${ }^{18}$ I. Furic, ${ }^{19}$ M. Gallinaro, ${ }^{52 a}$ J. Galyardt, ${ }^{13}$ F. Garberson, ${ }^{11}$ J. E. Garcia, ${ }^{21}$ A. F. Garfinkel, ${ }^{49}$ K. Genser,${ }^{18}$ H. Gerberich, ${ }^{25}$ D. Gerdes,${ }^{35}$ A. Gessler, ${ }^{27}$ S. Giagu, ${ }^{52 b, 52 a}$ V. Giakoumopoulou, ${ }^{3}$ P. Giannetti, ${ }^{47 a}$ K. Gibson, ${ }^{48}$ J. L. Gimmell, ${ }^{50}$ C. M. Ginsburg, ${ }^{18}$ N. Giokaris, ${ }^{3}$ M. Giordani, ${ }^{55 b, 55 a}$ P. Giromini, ${ }^{20}$ M. Giunta, ${ }^{47 b, 47 a}$ G. Giurgiu, ${ }^{26}$ V. Glagolev, ${ }^{16}$ D. Glenzinski, ${ }^{18}$ M. Gold, ${ }^{38}$ N. Goldschmidt, ${ }^{19}$ A. Golossanov, ${ }^{18}$ G. Gomez, ${ }^{12}$ G. Gomez-Ceballos,${ }^{33}$ M. Goncharov,${ }^{54}$ O. González, ${ }^{32}$ I. Gorelov, ${ }^{38}$ A. T. Goshaw, ${ }^{17}$ K. Goulianos, ${ }^{51}$ A. Gresele, ${ }^{44 b, 44 a}$ S. Grinstein, ${ }^{23}$ C. Grosso-Pilcher,${ }^{14}$ R. C. Group, ${ }^{18}$ U. Grundler, ${ }^{25}$ J. Guimaraes da Costa, ${ }^{23}$ Z. Gunay-Unalan, ${ }^{36}$ C. Haber, ${ }^{29}$ K. Hahn, ${ }^{33}$ S. R. Hahn, ${ }^{18}$ E. Halkiadakis, ${ }^{53}$ B.-Y. Han ${ }^{50}$ J. Y. Han, ${ }^{50}$ F. Happacher, ${ }^{20}$ K. Hara,${ }^{56}$ D. Hare, ${ }^{53}$ M. Hare, ${ }^{57}$ S. Harper, ${ }^{43}$ R. F. Harr, ${ }^{59}$ R. M. Harris, ${ }^{18}$ M. Hartz, ${ }^{48}$ K. Hatakeyama, ${ }^{51}$ C. Hays, ${ }^{43}$ M. Heck, ${ }^{27}$ A. Heijboer, ${ }^{46}$ J. Heinrich, ${ }^{46}$ C. Henderson, ${ }^{33}$ M. Herndon,${ }^{60}$ J. Heuser, ${ }^{27}$ S. Hewamanage, ${ }^{5}$ D. Hidas, ${ }^{17}$ C. S. Hill, ${ }^{11, d}$ D. Hirschbuehl, ${ }^{27}$ A. Hocker, ${ }^{18}$ S. Hou, ${ }^{1}$ M. Houlden, ${ }^{30}$ S.-C. Hsu, ${ }^{29}$

B. T. Huffman ${ }^{43}$ R. E. Hughes, ${ }^{40}$ U. Husemann,${ }^{36}$ M. Hussein, ${ }^{36}$ U. Husemann, ${ }^{61}$ J. Huston, ${ }^{36}$ J. Incandela, ${ }^{11}$ G. Introzzi, ${ }^{47 a}$ M. Iori, ${ }^{52 b, 52 a}$ A. Ivanov,${ }^{8}$ E. James, ${ }^{18}$ B. Jayatilaka, ${ }^{17}$ E. J. Jeon,${ }^{28}$ M. K. Jha, ${ }^{6 a}$ S. Jindariani, ${ }^{18}$ W. Johnson, ${ }^{8}$ M. Jones, ${ }^{49}$ K. K. Joo, ${ }^{28}$ S. Y. Jun, ${ }^{13}$ J. E. Jung, ${ }^{28}$ T. R. Junk, ${ }^{18}$ T. Kamon, ${ }^{54}$ D. Kar, ${ }^{19}$ P. E. Karchin, ${ }^{59}$ Y. Kato, ${ }^{42}$

R. Kephart, ${ }^{18}$ J. Keung, ${ }^{46}$ V. Khotilovich, ${ }^{54}$ B. Kilminster, ${ }^{18}$ D. H. Kim, ${ }^{28}$ H. S. Kim, ${ }^{28}$ H. W. Kim, ${ }^{28}$ J.E. Kim, ${ }^{28}$ M. J. Kim, ${ }^{20}$ S. B. Kim ${ }^{28}$ S. H. Kim,${ }^{56}$ Y. K. Kim, ${ }^{14}$ N. Kimura,${ }^{56}$ L. Kirsch, ${ }^{7}$ S. Klimenko, ${ }^{19}$ B. Knuteson, ${ }^{33}$ B. R. Ko, ${ }^{17}$ K. Kondo, ${ }^{58}$ D. J. Kong ${ }^{28}$ J. Konigsberg, ${ }^{19}$ A. Korytov, ${ }^{19}$ A. V. Kotwal, ${ }^{17}$ M. Kreps, ${ }^{27}$ J. Kroll, ${ }^{46}$ D. Krop, ${ }^{14}$ N. Krumnack, ${ }^{5}$ M. Kruse, ${ }^{17}$ V. Krutelyov, ${ }^{11}$ T. Kubo, ${ }^{56}$ T. Kuhr, ${ }^{27}$ N. P. Kulkarni, ${ }^{59}$ M. Kurata, ${ }^{56}$ S. Kwang, ${ }^{14}$ A. T. Laasanen, ${ }^{49}$ S. Lami, ${ }^{47 a}$ S. Lammel, ${ }^{18}$ M. Lancaster, ${ }^{31}$ R. L. Lander, ${ }^{8}$ K. Lannon, ${ }^{40, \mathrm{r}}$ A. Lath,${ }^{53}$ G. Latino, ${ }^{47 \mathrm{c}, 47 \mathrm{a}}$ I. Lazzizzera, ${ }^{44 \mathrm{~b}, 44 \mathrm{a}}{ }^{\text {T. LeCompte }}{ }^{2}$ E. Lee,${ }^{54}$ H. S. Lee, ${ }^{14}$ S. W. Lee,${ }^{54, t}$ S. Leone, ${ }^{47 a}$ J. D. Lewis, ${ }^{18}$ C.-S. Lin, ${ }^{29}$ J. Linacre, ${ }^{43}$ M. Lindgren, ${ }^{18}$ E. Lipeles, ${ }^{46}$

A. Lister, ${ }^{8}$ D. O. Litvintsev, ${ }^{18}$ C. Liu, ${ }^{48}$ T. Liu, ${ }^{18}$ N. S. Lockyer, ${ }^{46}$ A. Loginov, ${ }^{61}$ M. Loreti,${ }^{44 b, 44 a}$ L. Lovas, ${ }^{15}$

D. Lucchesi ${ }^{44 b, 44 a}$ C. Luci, ${ }^{52 b, 52 a}$ J. Lueck,${ }^{27}$ P. Lujan, ${ }^{29}$ P. Lukens, ${ }^{18}$ G. Lungu, ${ }^{51}$ L. Lyons, ${ }^{43}$ J. Lys, ${ }^{29}$ R. Lysak,${ }^{15}$

D. MacQueen, ${ }^{34}$ R. Madrak,${ }^{18}$ K. Maeshima, ${ }^{18}$ K. Makhoul,${ }^{33}$ T. Maki ${ }^{24}$ P. Maksimovic, ${ }^{26}$ S. Malde ${ }^{43}$ S. Malik ${ }^{31}$

G. Manca, ${ }^{30, f}$ A. Manousakis-Katsikakis, ${ }^{3}$ F. Margaroli, ${ }^{49}$ C. Marino, ${ }^{27}$ C. P. Marino, ${ }^{25}$ A. Martin, ${ }^{61}$ V. Martin, ${ }^{22, m}$

M. Martínez, ${ }^{4}$ R. Martínez-Ballarín, ${ }^{32}$ T. Maruyama, ${ }^{56}$ P. Mastrandrea, ${ }^{52 a}$ T. Masubuchi, ${ }^{56}$ M. Mathis, ${ }^{26}$ M. E. Mattson, ${ }^{59}$ P. Mazzanti, ${ }^{6 a}$ K. S. McFarland ${ }^{50}$ P. McIntyre, ${ }^{54}$ R. McNulty,${ }^{30, k}$ A. Mehta ${ }^{30}$ P. Mehtala, ${ }^{24}$ A. Menzione, ${ }^{47 a}$ P. Merkel, ${ }^{49}$

C. Mesropian ${ }^{51}$ T. Miao, ${ }^{18}$ N. Miladinovic, ${ }^{7}$ R. Miller, ${ }^{36}$ C. Mills,${ }^{23}$ M. Milnik,${ }^{27}$ A. Mitra, ${ }^{1}$ G. Mitselmakher, ${ }^{19}$

H. Miyake ${ }^{56}$ N. Moggi, ${ }^{6 a}$ C. S. Moon,${ }^{28}$ R. Moore, ${ }^{18}$ M. J. Morello, ${ }^{47 b, 47 a}$ J. Morlok, ${ }^{27}$ P. Movilla Fernandez, ${ }^{18}$ J. Mülmenstädt, ${ }^{29}$ A. Mukherjee, ${ }^{18}$ Th. Muller,${ }^{27}$ R. Mumford,${ }^{26}$ P. Murat,,${ }^{18}$ M. Mussini, ${ }^{6 b, 6 a}$ J. Nachtman, ${ }^{18}$ Y. Nagai, ${ }^{56}$ A. Nagano, ${ }^{56}$ J. Naganoma, ${ }^{56}$ K. Nakamura, ${ }^{56}$ I. Nakano, ${ }^{41}$ A. Napier, ${ }^{57}$ V. Necula, ${ }^{17}$ J. Nett, ${ }^{60}$ C. Neu, ${ }^{46, v}$ 
M. S. Neubauer, ${ }^{25}$ S. Neubauer, ${ }^{27}$ J. Nielsen, ${ }^{29, h}$ L. Nodulman, ${ }^{2}$ M. Norman,,${ }^{10}$ O. Norniella, ${ }^{25}$ E. Nurse,${ }^{31}$ L. Oakes, ${ }^{43}$ S. H. Oh ${ }^{17}$ Y. D. Oh ${ }^{28}$ I. Oksuzian, ${ }^{19}$ T. Okusawa,${ }^{42}$ R. Orava, ${ }^{24}$ S. Pagan Griso, ${ }^{44 b, 44 a}$ E. Palencia, ${ }^{18}$ V. Papadimitriou, ${ }^{18}$ A. Papaikonomou, ${ }^{27}$ A. A. Paramonov, ${ }^{14}$ B. Parks,${ }^{40}$ S. Pashapour, ${ }^{34}$ J. Patrick, ${ }^{18}$ G. Pauletta, ${ }^{55 b, 55 a}$ M. Paulini, ${ }^{13}$ C. Paus,${ }^{33}$ T. Peiffer, ${ }^{27}$ D. E. Pellett, ${ }^{8}$ A. Penzo,${ }^{55 a}$ T. J. Phillips, ${ }^{17}$ G. Piacentino, ${ }^{47 a}$ E. Pianori,${ }^{46}$ L. Pinera,${ }^{19}$ K. Pitts,${ }^{25}$ C. Plager, ${ }^{9}$ L. Pondrom, ${ }^{60}$ O. Poukhov, ${ }^{16, a}$ N. Pounder, ${ }^{43}$ F. Prakoshyn, ${ }^{16}$ A. Pronko, ${ }^{18}$ J. Proudfoot, ${ }^{2}$ F. Ptohos, ${ }^{18, j}$ E. Pueschel, ${ }^{13}$ G. Punzi ${ }^{47 b, 47 a}$ J. Pursley, ${ }^{60}$ J. Rademacker, ${ }^{43, d}$ A. Rahaman, ${ }^{48}$ V. Ramakrishnan, ${ }^{60}$ N. Ranjan, ${ }^{49}$ I. Redondo, ${ }^{32}$ P. Renton, ${ }^{43}$ M. Renz,${ }^{27}$ M. Rescigno, ${ }^{52 a}$ S. Richter, ${ }^{27}$ F. Rimondi, ${ }^{6 b, 6 a}$ L. Ristori, ${ }^{47 a}$ A. Robson, ${ }^{22}$ T. Rodrigo, ${ }^{12}$ T. Rodriguez, ${ }^{46}$

E. Rogers, ${ }^{25}$ S. Rolli,${ }^{57}$ R. Roser, ${ }^{18}$ M. Rossi,${ }^{55}$ R. Rossin, ${ }^{11}$ P. Roy, ${ }^{34}$ A. Ruiz,${ }^{12}$ J. Russ, ${ }^{13}$ V. Rusu, ${ }^{18}$ A. Safonov, ${ }^{54}$ W. K. Sakumoto, ${ }^{50}$ O. Saltó, ${ }^{4}$ L. Santi, ${ }^{55 b, 55 a}$ S. Sarkar, ${ }^{52 b, 52 a}$ L. Sartori, ${ }^{47 a}$ K. Sato, ${ }^{18}$ A. Savoy-Navarro, ${ }^{45}$ P. Schlabach, ${ }^{18}$ A. Schmidt, ${ }^{27}$ E. E. Schmidt, ${ }^{18}$ M. A. Schmidt, ${ }^{14}$ M. P. Schmidt,${ }^{61, a}$ M. Schmitt, ${ }^{39}$ T. Schwarz, ${ }^{8}$ L. Scodellaro, ${ }^{12}$

A. Scribano, ${ }^{47 c, 47 a}$ F. Scuri, ${ }^{47 a}$ A. Sedov,${ }^{49}$ S. Seidel,${ }^{38}$ Y. Seiya ${ }^{42}$ A. Semenov, ${ }^{16}$ L. Sexton-Kennedy, ${ }^{18}$ F. Sforza, ${ }^{47 a}$ A. Sfyrla, ${ }^{25}$ S. Z. Shalhout ${ }^{59}$ T. Shears, ${ }^{30}$ P. F. Shepard, ${ }^{48}$ M. Shimojima, ${ }^{56, q}$ S. Shiraishi,${ }^{14}$ M. Shochet,${ }^{14}$ Y. Shon, ${ }^{60}$

I. Shreyber, ${ }^{37}$ A. Sidoti, ${ }^{47 a}$ P. Sinervo, ${ }^{34}$ A. Sisakyan, ${ }^{16}$ A. J. Slaughter, ${ }^{18}$ J. Slaunwhite, ${ }^{40}$ K. Sliwa, ${ }^{57}$ J. R. Smith ${ }^{8}$

F. D. Snider ${ }^{18}$ R. Snihur, ${ }^{34}$ A. Soha, ${ }^{8}$ S. Somalwar,${ }^{53}$ V. Sorin, ${ }^{36}$ J. Spalding,${ }^{18}$ T. Spreitzer, ${ }^{34}$ P. Squillacioti, ${ }^{47 c, 47 a}$ M. Stanitzki, ${ }^{61}$ R. St. Denis, ${ }^{22}$ B. Stelzer,${ }^{34}$ O. Stelzer-Chilton, ${ }^{34}$ D. Stentz, ${ }^{39}$ J. Strologas,${ }^{38}$ G. L. Strycker, ${ }^{35}$ D. Stuart, ${ }^{11}$ J. S. Suh, ${ }^{28}$ A. Sukhanov, ${ }^{19}$ I. Suslov, ${ }^{16}$ T. Suzuki, ${ }^{56}$ A. Taffard, ${ }^{25, g}$ R. Takashima ${ }^{41}$ Y. Takeuchi, ${ }^{56}$ R. Tanaka, ${ }^{41}$ M. Tecchio, ${ }^{35}$ P. K. Teng, ${ }^{1}$ K. Terashi, ${ }^{51}$ J. Thom, ${ }^{18, i}$ A. S. Thompson, ${ }^{22}$ G. A. Thompson, ${ }^{25}$ E. Thomson,${ }^{46}$ P. Tipton, ${ }^{61}$ P. Ttito-Guzmán, ${ }^{32} \mathrm{~S}$. Tkaczyk, ${ }^{18} \mathrm{D}$. Toback,${ }^{54} \mathrm{~S}$. Tokar, ${ }^{15} \mathrm{~K}$. Tollefson, ${ }^{36} \mathrm{~T}$. Tomura, ${ }^{56} \mathrm{D}$. Tonelli, ${ }^{18} \mathrm{~S}$. Torre, ${ }^{20}$ D. Torretta, ${ }^{18}$ P. Totaro, ${ }^{55 b, 55 a}$ S. Tourneur, ${ }^{45}$ M. Trovato, ${ }^{47 a}$ S.-Y. Tsai, ${ }^{1}$ Y. Tu, ${ }^{46}$ N. Turini, ${ }^{47 c, 47 a}$ F. Ukegawa, ${ }^{56}$ S. Vallecorsa, ${ }^{21}$ N. van Remortel,${ }^{24, \mathrm{c}}$ A. Varganov, ${ }^{35}$ E. Vataga, ${ }^{47 \mathrm{~d}, 47 \mathrm{a}}$ F. Vázquez, ${ }^{19, \mathrm{n}} \mathrm{G}$. Velev, ${ }^{18} \mathrm{C}$. Vellidis, ${ }^{3}$ V. Veszpremi ${ }^{49}$ M. Vidal, ${ }^{32}$ R. Vidal,${ }^{18}$ I. Vila, ${ }^{12}$ R. Vilar, ${ }^{12}$ T. Vine, ${ }^{31}$ M. Vogel,${ }^{38}$ I. Volobouev, ${ }^{29, u}$ G. Volpi,${ }^{47 b, 47 a}$ P. Wagner, ${ }^{46}$ R. G. Wagner, ${ }^{2}$ R. L. Wagner, ${ }^{18}$ W. Wagner, ${ }^{27}$ J. Wagner-Kuhr, ${ }^{27}$ T. Wakisaka, ${ }^{42}$ R. Wallny, ${ }^{9}$ S. M. Wang, ${ }^{1}$ A. Warburton, ${ }^{34}$ D. Waters, ${ }^{31}$ M. Weinberger,${ }^{54}$ J. Weinelt, ${ }^{27}$ W. C. Wester III, ${ }^{18}$ B. Whitehouse,,${ }^{57}$ D. Whiteson, ${ }^{46, g}$ A. B. Wicklund, ${ }^{2}$ E. Wicklund, ${ }^{18}$ S. Wilbur, ${ }^{14}$ G. Williams, ${ }^{34}$ H. H. Williams, ${ }^{46}$ P. Wilson, ${ }^{18}$ B. L. Winer,${ }^{40}$ P. Wittich, ${ }^{18, i}$ S. Wolbers, ${ }^{18}$ C. Wolfe, ${ }^{14}$ T. Wright, ${ }^{35}$ X. Wu,${ }^{21}$ F. Würthwein, ${ }^{10}$ S. M. Wynne,${ }^{30}$ S. Xie, ${ }^{33}$ A. Yagil,${ }^{10}$ K. Yamamoto, ${ }^{42}$ J. Yamaoka, ${ }^{53}$ U. K. Yang, ${ }^{14, p}$ Y. C. Yang, ${ }^{28}$ W. M. Yao, ${ }^{29}$ G. P. Yeh, ${ }^{18}$ J. Yoh, ${ }^{18}$ K. Yorita, ${ }^{58}$ T. Yoshida,${ }^{42}$ G. B. Yu, ${ }^{50}$ I. Yu, ${ }^{28}$ S. S. Yu, ${ }^{18}$ J. C. Yun, ${ }^{18}$ L. Zanello, ${ }^{52 b, 52 a}$ A. Zanetti, ${ }^{55 a}$ X. Zhang, ${ }^{25}$ Y. Zheng,,${ }^{9, e}$ and S. Zucchelli ${ }^{6 b, 6 a}$

\section{(CDF Collaboration)}

${ }^{1}$ Institute of Physics, Academia Sinica, Taipei, Taiwan 11529, Republic of China

${ }^{2}$ Argonne National Laboratory, Argonne, Illinois 60439, USA

${ }^{3}$ University of Athens, 15771 Athens, Greece

${ }^{4}$ Institut de Fisica d'Altes Energies, Universitat Autonoma de Barcelona, E-08193, Bellaterra (Barcelona), Spain

${ }^{5}$ Baylor University, Waco, Texas 76798, USA

${ }^{6}$ Istituto Nazionale di Fisica Nucleare Bologna, I-40127 Bologna, Italy

${ }^{6 \mathrm{~b}}$ University of Bologna, I-40127 Bologna, Italy

${ }^{7}$ Brandeis University, Waltham, Massachusetts 02254, USA

${ }^{8}$ University of California, Davis, Davis, California 95616, USA

${ }^{9}$ University of California, Los Angeles, Los Angeles, California 90024, USA

${ }^{10}$ University of California, San Diego, La Jolla, California 92093, USA

${ }^{11}$ University of California, Santa Barbara, Santa Barbara, California 93106, USA

${ }^{12}$ Instituto de Fisica de Cantabria, CSIC-University of Cantabria, 39005 Santander, Spain

${ }^{13}$ Carnegie Mellon University, Pittsburgh, Pennsylvania 15213, USA

${ }^{14}$ Enrico Fermi Institute, University of Chicago, Chicago, Illinois 60637, USA

${ }^{15}$ Comenius University, 84248 Bratislava, Slovakia; Institute of Experimental Physics, 04001 Kosice, Slovakia

${ }^{16}$ Joint Institute for Nuclear Research, RU-141980 Dubna, Russia

${ }^{17}$ Duke University, Durham, North Carolina 27708, USA

${ }^{18}$ Fermi National Accelerator Laboratory, Batavia, Illinois 60510, USA

${ }^{19}$ University of Florida, Gainesville, Florida 32611, USA

${ }^{20}$ Laboratori Nazionali di Frascati, Istituto Nazionale di Fisica Nucleare, I-00044 Frascati, Italy

${ }^{21}$ University of Geneva, CH-1211 Geneva 4, Switzerland

${ }^{22}$ Glasgow University, Glasgow G12 8QQ, United Kingdom

${ }^{23}$ Harvard University, Cambridge, Massachusetts 02138, USA 
${ }^{24}$ Division of High Energy Physics, Department of Physics, University of Helsinki and Helsinki Institute of Physics, FIN-00014, Helsinki, Finland

${ }^{25}$ University of Illinois, Urbana, Illinois 61801, USA

${ }^{26}$ The Johns Hopkins University, Baltimore, Maryland 21218, USA

${ }^{27}$ Institut für Experimentelle Kernphysik, Universität Karlsruhe, 76128 Karlsruhe, Germany

${ }^{28}$ Center for High Energy Physics: Kyungpook National University, Daegu 702-701, Korea; Seoul National University, Seoul 151-742, Korea; Sungkyunkwan University, Suwon 440-746, Korea;

Korea Institute of Science and Technology Information, Daejeon, 305-806, Korea; Chonnam National University, Gwangju, 500-757, Korea

${ }^{29}$ Ernest Orlando Lawrence Berkeley National Laboratory, Berkeley, California 94720, USA

${ }^{30}$ University of Liverpool, Liverpool L69 7ZE, United Kingdom

${ }^{31}$ University College London, London WC1E 6BT, United Kingdom

${ }^{32}$ Centro de Investigaciones Energeticas Medioambientales y Tecnologicas, E-28040 Madrid, Spain

${ }^{33}$ Massachusetts Institute of Technology, Cambridge, Massachusetts 02139, USA

${ }^{34}$ Institute of Particle Physics: McGill University, Montréal, Québec, Canada H3A 2T8;

Simon Fraser University, Burnaby, British Columbia, Canada V5A 1S6; University of Toronto, Toronto, Ontario, Canada M5S 1A7; and TRIUMF, Vancouver, British Columbia, Canada V6T $2 A 3$

${ }^{35}$ University of Michigan, Ann Arbor, Michigan 48109, USA

${ }^{36}$ Michigan State University, East Lansing, Michigan 48824, USA

${ }^{37}$ Institution for Theoretical and Experimental Physics, ITEP, Moscow 117259, Russia

${ }^{38}$ University of New Mexico, Albuquerque, New Mexico 87131, USA

${ }^{39}$ Northwestern University, Evanston, Illinois 60208, USA

${ }^{40}$ The Ohio State University, Columbus, Ohio 43210, USA

${ }^{41}$ Okayama University, Okayama 700-8530, Japan

${ }^{42}$ Osaka City University, Osaka 588, Japan

${ }^{43}$ University of Oxford, Oxford OX1 3RH, United Kingdom

${ }^{44 a}$ Istituto Nazionale di Fisica Nucleare, Sezione di Padova-Trento, I-35131 Padova, Italy

${ }^{44 b}$ University of Padova, I-35131 Padova, Italy

${ }^{45}$ LPNHE, Universite Pierre et Marie Curie/IN2P3-CNRS, UMR7585, Paris, F-75252 France

${ }^{46}$ University of Pennsylvania, Philadelphia, Pennsylvania 19104, USA

${ }^{47 a}$ Istituto Nazionale di Fisica Nucleare Pisa, I-56127 Pisa, Italy

${ }^{47 \mathrm{~b}}$ University of Pisa, I-56127 Pisa, Italy

${ }^{47 c}$ University of Siena, I-56127 Pisa, Italy

${ }^{47 \mathrm{~d}}$ Scuola Normale Superiore, I-56127 Pisa, Italy

${ }^{48}$ University of Pittsburgh, Pittsburgh, Pennsylvania 15260, USA

${ }^{49}$ Purdue University, West Lafayette, Indiana 47907, USA

${ }^{50}$ University of Rochester, Rochester, New York 14627, USA

${ }^{51}$ The Rockefeller University, New York, New York 10021, USA

${ }^{52 \mathrm{a}}$ Istituto Nazionale di Fisica Nucleare, Sezione di Roma 1, I-00185 Roma, Italy

${ }^{52 \mathrm{~b}}$ Sapienza Università di Roma, I-00185 Roma, Italy

${ }^{53}$ Rutgers University, Piscataway, New Jersey 08855, USA

${ }^{54}$ Texas A\&M University, College Station, Texas 77843, USA

${ }^{55 a}$ Istituto Nazionale di Fisica Nucleare Trieste/Udine, Italy

${ }^{55 \mathrm{~b}}$ University of Trieste/Udine, Italy

${ }^{56}$ University of Tsukuba, Tsukuba, Ibaraki 305, Japan

${ }^{57}$ Tufts University, Medford, Massachusetts 02155, USA

${ }^{58}$ Waseda University, Tokyo 169, Japan

${ }^{59}$ Wayne State University, Detroit, Michigan 48201, USA

${ }^{60}$ University of Wisconsin, Madison, Wisconsin 53706, USA

${ }^{61}$ Yale University, New Haven, Connecticut 06520, USA

(Received 4 November 2008; published 6 March 2009)

We present a search for high-mass neutral resonances using dimuon data corresponding to an integrated luminosity of $2.3 \mathrm{fb}^{-1}$ collected in $p \bar{p}$ collisions at $\sqrt{s}=1.96 \mathrm{TeV}$ by the CDF II detector at the Fermilab Tevatron. No significant excess above the standard model expectation is observed in the dimuon invariantmass spectrum. We set $95 \%$ confidence level upper limits on $\sigma \operatorname{BR}(p \bar{p} \rightarrow X \rightarrow \mu \bar{\mu})$, where $X$ is a boson with spin- 0,1 , or 2 . Using these cross section limits, we determine lower mass limits on sneutrinos in $R$-parity-violating supersymmetric models, $Z^{\prime}$ bosons, and Kaluza-Klein gravitons in the RandallSundrum model. 
DOI: 10.1103/PhysRevLett.102.091805

Neutral resonances decaying to muons have historically been a source of major discoveries. They also occur in a variety of theoretical models which attempt to unify the standard model (SM) forces or explain the large gap between the SM and gravitational energy scales. The gauge group $S U(3)_{C} \times S U(2)_{L} \times U(1)_{Y}$ of the $\mathrm{SM}$ can be embedded in larger gauge groups such as $S U(5), S O(10)$, and $E_{6}$, to achieve unification in a grand unified theory [1-4]. In many schemes of grand unified theory symmetry breaking, $U(1)$ gauge groups survive to relatively low energies [2], leading to the prediction of neutral gauge vector $\left(Z^{\prime}\right)$ bosons. Such $Z^{\prime}$ bosons typically couple with electroweak strength to SM fermions, and can be observed at hadron colliders as narrow, spin-1, dimuon resonances from $q \bar{q} \rightarrow$ $Z^{\prime} \rightarrow \mu \bar{\mu}$. Many other models, such as the $S U(2)_{L} \times$ $S U(2)_{R} \times U(1)_{B-L}$ gauge group of the left-right model [5], and the "little Higgs" models [6,7], also predict heavy neutral gauge bosons.

Additional spatial dimensions are a possible explanation for the gap between the electroweak symmetry-breaking scale and the gravitational energy scale $M_{\text {Planck }}[8,9]$. The Randall-Sundrum (RS) model [9] predicts excited KaluzaKlein modes of the graviton, which appear as spin-2 resonances $G^{*}$ in the process $q \bar{q} \rightarrow G^{*} \rightarrow \mu \bar{\mu}$. These modes have a narrow intrinsic width when $k / M_{\text {Planck }}<0.1$, where $k^{2}$ is the spacetime curvature in the extra dimension. In superstring theories with $\mathcal{O}(1)$ couplings, $k / M_{\text {Planck }} \approx$ $0.01[10]$.

Spin-0 resonances such as the sneutrino $\tilde{\nu}$ in the process $q \bar{q} \rightarrow \tilde{\nu}, \tilde{\bar{\nu}} \rightarrow \mu \bar{\mu}$ are predicted by supersymmetric theories with $R$-parity violation [11]. Scalar Higgs bosons can be produced as resonances and decay to dimuons.

The most sensitive direct searches for high-mass boson resonances, which have previously been performed at the Tevatron, have set 95\% confidence level (C.L.) lower limits on the masses $M_{Z^{\prime}}, M_{G^{*}}$, and $M_{\tilde{\nu}}$ of $Z^{\prime}$ bosons, RS gravitons, and sneutrinos, respectively. The previous dimuon publication from CDF II, based on $\approx 200 \mathrm{pb}^{-1}$ of integrated luminosity [12], set mass limits that vary from 170 to $885 \mathrm{GeV}$ [13] depending on the boson spin and couplings to the SM fermions. Other dilepton and diphoton decay channels have also been explored at the Tevatron $[14,15]$. Using an order of magnitude more data, we present in this Letter the most sensitive direct search to date for $Z^{\prime}, G^{*}$, and $\tilde{\nu}$ bosons at high mass.

This analysis uses $2.3 \mathrm{fb}^{-1}$ of data from $p \bar{p}$ collisions at $\sqrt{s}=1.96 \mathrm{TeV}$ in the CDF II detector $[16,17]$. CDF II is a magnetic spectrometer surrounded by calorimeters and muon detectors. We use the central drift chamber (COT) [18], the central calorimeter [19], and the muon detectors [20] for identification and measurement of muons with $|\eta|<1$ [13]. The online selection requires a COT track with $p_{T}>18 \mathrm{GeV}$ [13], and matching muon detector hits.
PACS numbers: $13.85 . \mathrm{Rm}, 12.60 . \mathrm{Cn}, 13.85 . \mathrm{Qk}, 14.70 . \mathrm{Pw}$

We select a pair of oppositely charged muons, each with a COT track with $p_{T}>30 \mathrm{GeV}$ passing quality requirements, and a minimum-ionization signal in the calorimeter. Cosmic rays are rejected using COT hit timing [21]. The dimuon signal sample consists of 68150 events in the control region $70<m_{\mu \bar{\mu}}<100 \mathrm{GeV}$, where the $p \bar{p} \rightarrow$ $Z \rightarrow \mu \bar{\mu}$ process dominates, and 3804 events in the search region $m_{\mu \bar{\mu}}>100 \mathrm{GeV}$.

The alignment of the COT is performed using a pure sample of high-momentum cosmic-ray muons, in order to obtain the best possible dimuon mass resolution. Each muon's complete trajectory is fitted to a single helix [21]. The fits are used to determine the relative locations of the sense wires, including gravitational and electrostatic displacements, with a statistical accuracy of a few microns [17]. We constrain remaining misalignments, which cause a bias in the track curvature, by comparing $\langle E / p\rangle$ [13] for electrons and positrons. The tracker momentum scale and resolution are measured by template fitting the $Z \rightarrow \mu \bar{\mu}$ mass peak, and calibrating to the world average values [22] of the $Z$ boson mass and width.

For a resonance with electroweak coupling and mass above $200 \mathrm{GeV}$, the observed width of the $m_{\mu \bar{\mu}}$ distribution is dominated by the track curvature resolution, resulting in an approximately constant resolution of $\delta m_{\mu \bar{\mu}}^{-1} \approx$ $0.17 \mathrm{TeV}^{-1}$. Our search strategy is to construct templates of the observable $m_{\mu \bar{\mu}}^{-1}$ distribution for a range of boson Breit-Wigner pole masses, add the background distributions to the templates, and compare the templates to the $m_{\mu \bar{\mu}}^{-1}$ distribution from the data in the search region $m_{\mu \bar{\mu}}>$ $100 \mathrm{GeV}$. The simulated templates (including backgrounds) are normalized to the data in the $70<m_{\mu \bar{\mu}}<$ $100 \mathrm{GeV}$ region, thus canceling several sources of systematic uncertainty.

We determine the most likely number of signal events $\left(N_{S}\right)$, and the corresponding confidence intervals [23], from the binned Poisson likelihood [17] for the observed data to be produced by a sum of signal and background templates. The use of the constant-resolution variable $m_{\mu \bar{\mu}}^{-1}$ simplifies the optimization of the template binning and the scan over the boson pole masses.

Signal and SM Drell-Yan background distributions are evaluated using a specialized Monte Carlo simulation [17] of boson production and decay, and of the detector response to the leptons and hadrons. The kinematics of boson production and decay are obtained from the PYTHIA [24] event generator using the CTEQ6M [25] set of parton distribution functions. QED radiation is simulated [17] based on the WGRAD program [26]. The Monte Carlo program performs a detailed hit-level simulation of the lepton tracks. COT hits are generated according to their resolution $(\approx 150 \mu \mathrm{m})$ and measured efficiencies, and a helix fit is 


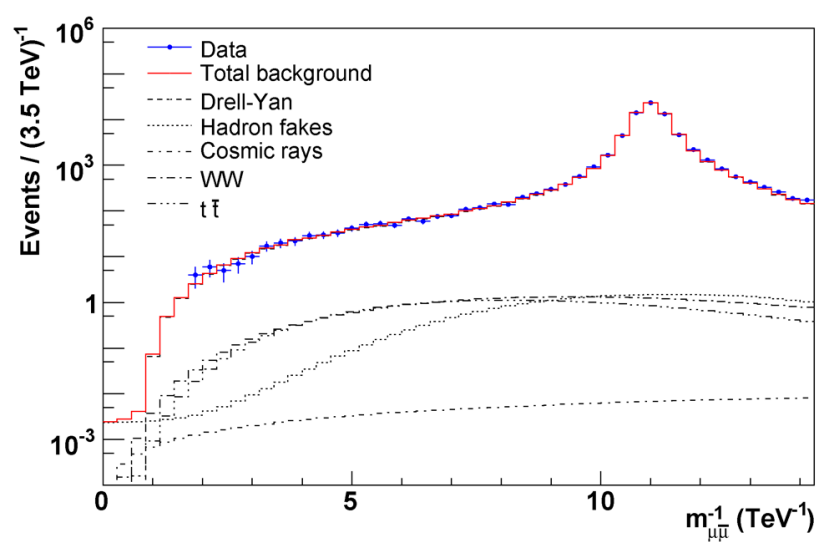

FIG. 1 (color online). The distribution of $m_{\mu \bar{\mu}}^{-1}\left(\mathrm{TeV}^{-1}\right)$ for the observed data (points), the individual backgrounds (dotted or dashed histograms) and the summed background (solid histogram). The $Z$ boson peak is prominently seen. The inverse mass distribution has the useful feature that the detector resolution is constant $\left(\approx 0.17 \mathrm{TeV}^{-1}\right)$ over the range shown in the plot.

performed (as it is in data) to simulate the reconstructed track. We apply a mass-dependent next-to-next-to-leading order (NNLO) multiplicative correction ( $K$ factor) [27] to the SM Drell-Yan background.

The SM production processes for $W^{+} W^{-}[28]$ and $t \bar{t}$ [29] have small contributions, and are evaluated using their NLO cross sections, PYTHIA, and a detector simulation based on GEANT [30]. Misidentification backgrounds result from cosmic rays, QCD jets, and $\pi / K$ decays in flight (DIF). We evaluate the cosmic-ray background using a large sample of cosmic rays identified with the COT-timing-based algorithm [21], and using the direction-of-flight information provided by this algorithm. The $m_{\mu \bar{\mu}}^{-1}$ shape of misidentified jets is evaluated from a large sample of inclusive jet events. Decays in flight within the COT active volume generate a kink along the helical trajectory, resulting in a mismeasurement of the track curvature. For large reconstructed momenta, the measured DIF curvature distribution is approximately uniform and leads to a flat $m_{\mu \bar{\mu}}^{-1}$ spectrum. Most DIF tracks are rejected using their abnormal COT-hit pattern and large fit $\chi^{2}$. The jet and DIF backgrounds are normalized using the mass distribution of same-charge dimuon events.

Figure 1 shows the $m_{\mu \mu}^{-1}$ distributions of the observed data and the expected backgrounds, which are in good agreement (as shown in Fig. 2). A resonance whose observed width is dominated by detector resolution would appear as a peak spanning approximately three bins. The likelihood-based fitter finds no significant excess. We use background-only ensembles of simulated events, each with the statistics of the data sample, to evaluate the probability of statistical fluctuations anywhere in the search region generating a discrepancy at least as significant as the largest discrepancy found in the data. We find this proba-

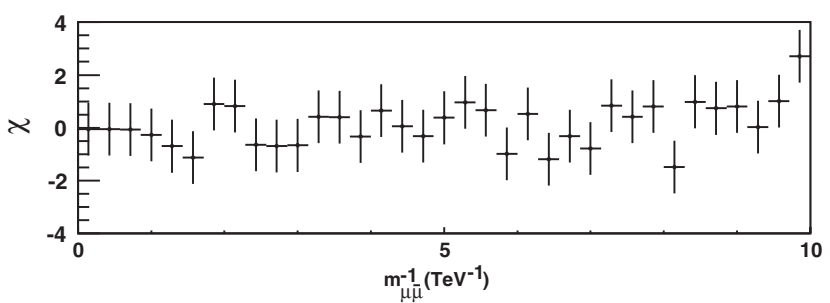

FIG. 2. The difference between the distributions of $m_{\mu \bar{\mu}}^{-1}$ $\left(\mathrm{TeV}^{-1}\right)$ for the observed data and the summed background, divided by the expected statistical uncertainty in each bin. All vertical error bars have unit size.

bility (" $p$ value") to be $6.6 \%$ and we conclude that the observed data are statistically consistent with the SM expectation. The dielectron $m_{e e}$ spectrum from $2.5 \mathrm{fb}^{-1}$ of CDF II data [31] shows that the largest discrepancy with the expected background occurs at $m_{e e} \sim 240 \mathrm{GeV}$. Figure 2 shows that the dimuon data are consistent with the expectation near this mass to better than $1 \sigma$ in statistical precision. The sensitivity of the dielectron analysis for a spin- 1 resonance at this mass is $\approx 20 \%$ better than the dimuon analysis reported here.

The likelihood fitter determines the 95\% C.L. upper limit on the number of signal events, for each value of the resonance pole mass. We convert these limits to limits on $\sigma \mathrm{BR}(\tilde{\nu}, \tilde{\bar{\nu}} \rightarrow \mu \bar{\mu}), \sigma \mathrm{BR}\left(Z^{\prime} \rightarrow \mu \bar{\mu}\right)$, and $\sigma \operatorname{BR}\left(G^{*} \rightarrow\right.$ $\mu \bar{\mu})$ using the total acceptance as a function of pole mass, the NNLO cross section for $Z \rightarrow \mu \mu$ of $251.3 \mathrm{pb}$ [16], and dividing by the observed number of $Z \rightarrow \mu \bar{\mu}$ events. The acceptance is verified with the detailed GEANTbased simulation, and comparisons to data distributions. The muon identification efficiency is verified using a pure data sample of $Z$ bosons triggered by one identified muon. The total acceptance, including kinematic and fiducial acceptance and dimuon identification, increases from $\approx 13 \%(\approx 20 \%)$ for a pole mass of $90 \mathrm{GeV}$ to $\approx 40 \%$ $\left(\approx 45 \%\right.$ ) for a $Z^{\prime}$ (graviton) pole mass of $1 \mathrm{TeV}$, and decreases for higher pole masses due to the kinematic limit of the parton collisions. The 95\% C.L. upper limits on $\sigma \mathrm{BR}(\tilde{\nu}, \tilde{\bar{\nu}} \rightarrow \mu \bar{\mu}), \sigma \mathrm{BR}\left(Z^{\prime} \rightarrow \mu \bar{\mu}\right)$, and $\sigma \mathrm{BR}\left(G^{*} \rightarrow \mu \bar{\mu}\right)$ are shown in Fig. 3. The dominant mass-dependent systematic uncertainties arise from parton distribution functions $(16 \%)$, the NNLO $K$ factor (9\%) [27], QED radiative corrections (3\%) [32], and acceptance (3\%), all quoted at $1 \mathrm{TeV}$. These uncertainties are incorporated as functions of $m_{\mu \bar{\mu}}$ and increase monotonically beyond $100 \mathrm{GeV}$. Uncertainties on the momentum scale and resolution, and on the non-Drell-Yan background predictions, have a negligible effect.

Our signal templates have been generated with a resonance pole width $\Gamma=2.8 \% \times M$, based on the SM $Z$ boson width. Thus our signal scan probes an observed width of $\approx[17 \%(M / \mathrm{TeV}) \oplus 2.8 \%] M$. In a model where the observed width increases by a factor $x$, the cross section limits would increase by about a factor of $\sqrt{x}$. 

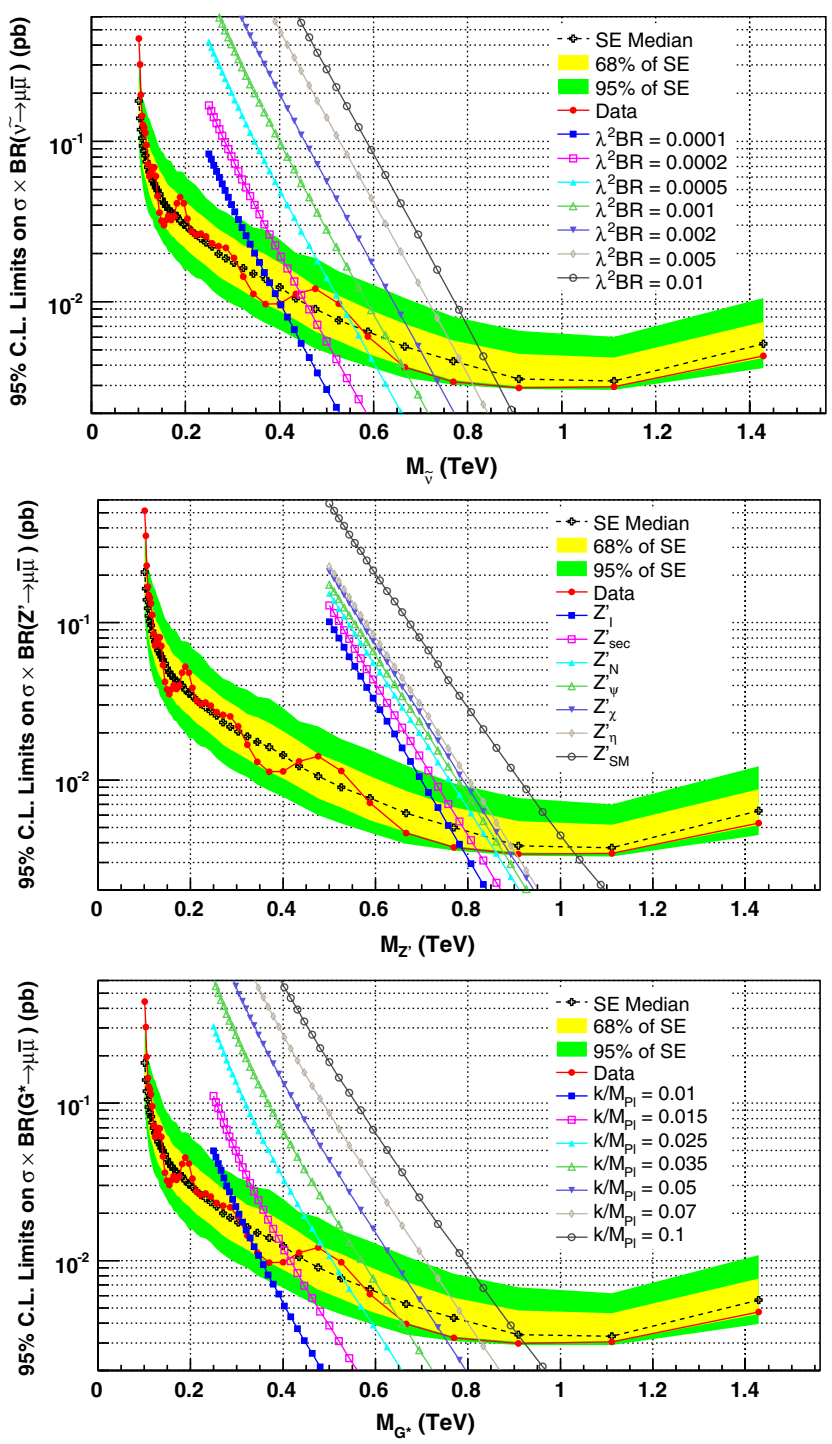

FIG. 3 (color online). The 95\% C.L. upper limits on $\sigma \mathrm{BR}(\tilde{\nu}, \tilde{\bar{\nu}} \rightarrow \mu \bar{\mu}) \quad$ vs $\quad M_{\tilde{\nu}}$ (top), $\sigma \mathrm{BR}\left(Z^{\prime} \rightarrow \mu \bar{\mu}\right) \quad$ vs $\quad M_{Z^{\prime}}$ (middle), and $\sigma \mathrm{BR}\left(G^{*} \rightarrow \mu \bar{\mu}\right)$ vs $M_{G^{*}}$ (bottom). Also shown are the theoretical cross sections for various model parameter values $[9,11,33]$. The expected limits and ranges of limits, as derived from simulated experiments (SE), are shown for comparison. The step size between adjacent templates in the signal scan is $0.2 \mathrm{TeV}^{-1}$ in pole mass.

We use PYTHIA to compute the cross sections for production of $Z^{\prime}$ bosons predicted by $E_{6}$ models [33] or having the same couplings to SM fermions as the $Z$ boson, and of $G^{*}$ bosons for various $k / M_{\text {Planck }}$ values. We apply the NNLO $K$ factor to these leading order cross sections. The NLO $\tilde{\nu}$ production cross sections are obtained from [11]. We derive the boson mass limits shown in Table I.

In conclusion, we have presented a direct search for high-mass neutral resonances with spin- 0,1 , and 2 , using an integrated luminosity of $2.3 \mathrm{fb}^{-1}$ collected by the CDF II detector. Our dimuon invariant-mass spectrum is
TABLE I. 95\% C.L. lower limits on $Z^{\prime}$, graviton, and sneutrino masses (in $\mathrm{GeV}$ ) for various model parameters [9,11,33]. For the $R$-parity-violating sneutrino model, $\lambda$ is the $d \bar{d} \tilde{\nu}$ coupling, and BR denotes the $\tilde{\nu}, \tilde{\bar{\nu}} \rightarrow \mu \bar{\mu}$ branching ratio.

\begin{tabular}{lccccc}
\hline \hline $\begin{array}{c}Z^{\prime} \\
\text { Model }\end{array}$ & $\begin{array}{c}Z^{\prime} \\
\text { Mass limit }\end{array}$ & $\begin{array}{c}\text { RS graviton } \\
k / M_{\text {Planck }}\end{array}$ & $\begin{array}{c}\text { Graviton } \\
\text { Mass limit }\end{array}$ & $\begin{array}{c}\tilde{\nu} \\
\lambda^{2} \mathrm{BR}\end{array}$ & $\begin{array}{c}\tilde{\nu} \\
\text { Mass limit }\end{array}$ \\
\hline$Z_{I}^{\prime}$ & 789 & 0.01 & 293 & 0.0001 & 397 \\
$Z_{\text {sec }}^{\prime}$ & 821 & 0.015 & 409 & 0.0002 & 441 \\
$Z_{N}^{\prime}$ & 861 & 0.025 & 493 & 0.0005 & 541 \\
$Z_{\psi}^{\prime}$ & 878 & 0.035 & 651 & 0.001 & 662 \\
$Z_{X}^{\prime}$ & 892 & 0.05 & 746 & 0.002 & 731 \\
$Z_{\eta}^{\prime}$ & 904 & 0.07 & 824 & 0.005 & 810 \\
$Z_{\text {SM }}^{\prime}$ & 1030 & 0.1 & 921 & 0.01 & 866 \\
\hline \hline
\end{tabular}

consistent with the SM expectation. We set the world's tightest constraints on $Z^{\prime}$ bosons in various models, on Kaluza-Klein graviton modes in the RS model, and on sneutrinos in $R$-parity-violating supersymmetric models. At $95 \%$ C.L., we exclude $100<M_{Z^{\prime}}<982 \mathrm{GeV}$ for a $Z_{\eta}^{\prime}$ boson of the $E_{6}$ model, $100<M_{G^{*}}<921 \mathrm{GeV}$ for $k / M_{\text {Planck }}=0.1, \quad$ and $\quad 100<M_{\tilde{\nu}}<810 \mathrm{GeV}$ for $\lambda^{2} \operatorname{BR}(\tilde{\nu}, \tilde{\bar{\nu}} \rightarrow \mu \bar{\mu})=0.01$, where $\lambda$ is the $d \bar{d} \tilde{\nu}$ coupling and BR denotes the $\tilde{\nu}, \tilde{\bar{\nu}} \rightarrow \mu \bar{\mu}$ branching ratio.

We thank the Fermilab staff and the technical staffs of the participating institutions for their vital contributions. This work was supported by the U.S. Department of Energy and National Science Foundation; the Italian Istituto Nazionale di Fisica Nucleare; the Ministry of Education, Culture, Sports, Science and Technology of Japan; the Natural Sciences and Engineering Research Council of Canada; the National Science Council of the Republic of China; the Swiss National Science Foundation; the A.P. Sloan Foundation; the Bundesministerium für Bildung und Forschung, Germany; the Korean Science and Engineering Foundation and the Korean Research Foundation; the Science and Technology Facilities Council and the Royal Society, U.K.; the Institut National de Physique Nucleaire et Physique des Particules/CNRS; the Russian Foundation for Basic Research; the Ministerio de Ciencia e Innovación, Spain; the Slovak R\&D Agency; and the Academy of Finland.

\footnotetext{
${ }^{\mathrm{a}}$ Deceased.

${ }^{\mathrm{b}}$ Visitor from University of Massachusetts Amherst, Amherst, MA 01003, USA.

${ }^{\mathrm{c}}$ Visitor from Universiteit Antwerpen, B-2610 Antwerp, Belgium.

${ }^{\mathrm{d}}$ Visitor from University of Bristol, Bristol BS8 1TL, United Kingdom.

${ }^{\mathrm{e}}$ Visitor from Chinese Academy of Sciences, Beijing 100864, China.

${ }^{\mathrm{f}}$ Visitor from Istituto Nazionale di Fisica Nucleare, Sezione di Cagliari, 09042 Monserrato (Cagliari), Italy.
} 
${ }^{\mathrm{g}}$ Visitor from University of California Irvine, Irvine, CA 92697, USA.

${ }^{\text {h}}$ Visitor from University of California Santa Cruz, Santa Cruz, CA 95064, USA.

${ }^{\mathrm{i}}$ Visitor from Cornell University, Ithaca, NY 14853, USA. ${ }^{j}$ Visitor from University of Cyprus, Nicosia CY-1678, Cyprus.

${ }^{\mathrm{k}}$ Visitor from University College Dublin, Dublin 4, Ireland.

${ }^{1}$ Visitor from Royal Society of Edinburgh, Edinburgh EH2 2PQ, United Kingdom.

${ }^{\mathrm{m}}$ Visitor from University of Edinburgh, Edinburgh EH9 3JZ, United Kingdom.

${ }^{\mathrm{n}}$ Visitor from Universidad Iberoamericana, Mexico D.F., Mexico.

${ }^{\circ}$ Visitor from Queen Mary, University of London, London, E1 4NS, England.

${ }^{\mathrm{p}}$ Visitor from University of Manchester, Manchester M13 9PL, England.

${ }^{\mathrm{q}}$ Visitor from Nagasaki Institute of Applied Science, Nagasaki, Japan.

${ }^{\mathrm{r}}$ Visitor from University of Notre Dame, Notre Dame, IN 46556, USA.

${ }^{\mathrm{s}}$ Visitor from University de Oviedo, E-33007 Oviedo, Spain.

${ }^{\mathrm{t}}$ Visitor from Texas Tech University, Lubbock, TX 79409, USA.

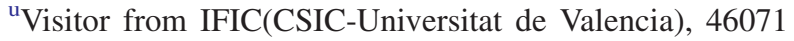
Valencia, Spain.

${ }^{\vee}$ Visitor from University of Virginia, Charlottesville, VA 22904, USA.

${ }^{\mathrm{w}}$ On leave from J. Stefan Institute, Ljubljana, Slovenia.

[1] J. L. Rosner, Phys. Rev. D 35, 2244 (1987).

[2] F. del Aguila, M. Quiros, and F. Zwirner, Nucl. Phys. B287, 419 (1987); J. L. Hewett and T. G. Rizzo, Phys. Rep. 183, 193 (1989).

[3] J. Erler et al., Phys. Rev. D 66, 015002 (2002); T. Han et al., Phys. Rev. D 70, 115006 (2004).

[4] J. Kang and P. Langacker, Phys. Rev. D 71, 035014 (2005).

[5] R. N. Mohapatra and J.C. Pati, Phys. Rev. D 11, 566 (1975); G. Senjanovic and R. N. Mohapatra, Phys. Rev. D 12, 1502 (1975); R. N. Mohapatra and G. Senjanovic, Phys. Rev. D 23, 165 (1981).

[6] N. Arkani-Hamed et al., J. High Energy Phys. 07 (2002) 034.

[7] T. Han et al., Phys. Rev. D 67, 095004 (2003).

[8] N. Arkani-Hamed, S. Dimopoulos, and G. Dvali, Phys. Lett. B 429, 263 (1998).

[9] L. Randall and R. Sundrum, Phys. Rev. Lett. 83, 4690 (1999); L. Randall and R. Sundrum, Phys. Rev. Lett. 83, 3370 (1999).

[10] H. Davoudiasl, J. L. Hewett, and T. G. Rizzo, Phys. Rev. Lett. 84, 2080 (2000).

[11] D. Choudhury, S. Majhi, and V. Ravindran, Nucl. Phys. B660, 343 (2003); We assume the $\tilde{\nu}$ and $\tilde{\bar{\nu}}$ have equal masses and couplings and contribute equally to a potential signal, as in W. Shao-Ming, H. Liang, M. Wen-Gan, Z. Ren-You, and J. Yi, Phys. Rev. D 74, 057902 (2006).

[12] A. Abulencia et al. (CDF Collaboration), Phys. Rev. Lett. 95, 252001 (2005).

[13] We use the convention $\hbar=c=1$. Pseudorapidity is defined as $\eta=-\ln [\tan (\theta / 2)]$, where $\theta$ is the polar angle from the beam axis. Track momentum $p$ transverse to the beam is denoted $p_{T}$. E denotes the calorimeter energy.

[14] T. Aaltonen et al. (CDF Collaboration), Phys. Rev. Lett. 99, 171802 (2007); T. Aaltonen et al. (CDF Collaboration), Phys. Rev. Lett. 99, 171801 (2007); A. Abulencia et al. (CDF Collaboration), Phys. Rev. Lett. 96, 211801 (2006); D. Acosta et al. (CDF Collaboration), Phys. Rev. Lett. 95, 131801 (2005).

[15] V. M. Abazov et al. (D0 Collaboration), Phys. Rev. Lett. 100, 091802 (2008).

[16] A. Abulencia et al. (CDF Collaboration), J. Phys. G 34, 2457 (2007); D. Acosta et al. (CDF Collaboration), Phys. Rev. Lett. 94, 091803 (2005).

[17] T. Aaltonen et al. (CDF Collaboration), Phys. Rev. D 77, 112001 (2008).

[18] T. Affolder et al., Nucl. Instrum. Methods Phys. Res., Sect. A 526, 249 (2004).

[19] F. Abe et al. (CDF Collaboration), Nucl. Instrum. Methods Phys. Res., Sect. A 271, 387 (1988).

[20] G. Ascoli et al., Nucl. Instrum. Methods Phys. Res., Sect. A 268, 33 (1988).

[21] A. V. Kotwal, H. K. Gerberich, and C. Hays, Nucl. Instrum. Methods Phys. Res., Sect. A 506, 110 (2003).

[22] S. Schael et al. (ALEPH, DELPHI, L3, OPAL, and SLD Collaborations), Phys. Rep. 427, 257 (2006), and references therein.

[23] G. J. Feldman and R. D. Cousins, Phys. Rev. D 57, 3873 (1998).

[24] T. Sjöstrand, Comput. Phys. Commun. 82, 74 (1994). We use PYTHIA version 6.208.

[25] J. Pumplin et al., J. High Energy Phys. 07 (2002) 012.

[26] U. Baur, S. Keller, and D. Wackeroth, Phys. Rev. D 59, 013002 (1998).

[27] M. Carena, A. Daleo, B. A. Dobrescu, and T. M.P. Tait, Phys. Rev. D 70, 093009 (2004).

[28] J. M. Campbell and R. K. Ellis, Phys. Rev. D 60, 113006 (1999). We use MCFM version 3.4.5 from mcfm.fnal.gov.

[29] R. Bonciani, S. Catani, M. L. Mangano, and P. Nason, Nucl. Phys. B529, 424 (1998); M. Cacciari et al., J. High Energy Phys. 04 (2004) 068.

[30] R. Brun and F. Carminati, CERN Program Library Long Writeup, W5013, 1993, version 3.15 (unpublished).

[31] T. Aaltonen et al. (CDF Collaboration), Phys. Rev. Lett. 102, 031801 (2009).

[32] U. Baur, O. Brein, W. Hollik, C. Schappacher, and D. Wackeroth, Phys. Rev. D 65, 033007 (2002), Fig. 3(b).

[33] The couplings we use are detailed in C. Ciobanu et al., FERMILAB Report No. FERMILAB-FN-0773-E, 2008. 